\title{
Study of The Trilinear Neighborhood Model of Process of Formation of Temperature's Coiling Hot-Rolled Strip
}

\author{
A. M. Shmyrin, A. G. Yurtsev, A. M. Korneev, L. S. Abdullakh
}

Lipetsk state technical University, Russia

\section{Article Info \\ Article history: \\ Received Feb 10, 2016 \\ Revised Apr 6, 2016 \\ Accepted Apr 20, 2016}

\section{Keyword:}

Extremums

Hot-rolled strip

Identification

Mixed management

Neighborhood system

Normalization

Parametric variables

\begin{abstract}
The paper considers trilinear neighborhood model of process of formation of temperature's coiling hot-rolled strip, where the parameters are the state, control and information. The methods of determining the composition of extremums of the general parametric operation are presented. Extremums' condition for the existence, which has been tested on a concrete example, is obtained. The assumption about the area in which it is impossible to say with certainty about the system's stability is stated. The hypothesis about the condition of the loss of the position of stable equilibrium of the system and the transition to a new state.
\end{abstract}

Copyright $@ 2016$ Institute of Advanced Engineering and Science. All rights reserved.

\section{Corresponding Author:}

Abdullakh L. S.,

Lipetsk state technical University, Russia,

30 Moskovskaya St.398600 Lipetsk, Russia.

Email: d.48@ rambler.ru

\section{INTRODUCTION}

Neighborhood models are a promising direction in the sphere of complex systems' modeling, since the differ with their description versatility with the help of the ambits of the bonds' structure between the system's nodes. Neighborhood systems' theory is a universal means of a category of discrete distributed systems modeling: stationary and dynamic, clear and illegible, with linear and nonlinear relationships. This theory is a generalizing for many discrete distributed systems, the neighborhood approach's use in application tasks covers systems of different nature and direction - from technical to economics. Neighborhood models - an extremely efficient modeling method which allows to reproduce complex dependencies. As an example, parametric neighborhood modeling of the process of formation of temperature's coiling hot-rolled strip. Microstructure of hot-rolled steel depends on the rolling's end temperature and coiling's temperature. To get a fine-grained uniform of the metal's structure of low carbon steel, its rolling must be finished at a temperature of 760-900 C, and the roll must be reeled at coiling's temperature of 540-720C. Strip's cooling after finishing group stands is realized on the deferent roller. The deferent roller of hot rolling mill is a significant part of the way through which the strip passes after rolling to reels. The main function of deferent roller's section is cooling the strip from the rolling temperature to a temperature suitable for the coiling. Above and below the strip, moving on the roller, tanks with water for the cooling are mounted. Tanks are united in half-section - the minimum controlled devices for cooling. In technological object's real functioning condition, the mathematical modeling is an effective method of indirectly finding the object's characteristics and its elements, its state and the relationships between them. Model's development of technological process and its control systems allows to identify additional characteristics and the object's attributes and thereby improve the management's quality. 


\section{RESEARCH METHOD}

Let's consider the realization of the method of the trilinear neighborhood model's construction on the complex distributed object's example - technological process of accelerated cooling of the hot strip on the broadband hot rolling mill.

In general form, trilinear neighborhood model looks like the following:

$$
\begin{aligned}
& \sum_{\alpha \in O_{x}[a]} w_{x}[a, \alpha] X[\alpha]+\sum_{\beta \in O_{v}[a]} w_{v}[a, \beta] V[\beta]+\sum_{\gamma \in O_{y}[a]} w_{y}[a, \gamma] Y[\gamma]+ \\
& +\sum_{\alpha \in O_{x}[a] \beta \in O_{v}[a]} w_{x v}[a, \alpha, \beta] X[\alpha] V[\beta]+\sum_{\alpha \in O_{x}[a]} \sum_{\gamma \in O_{y}[a]} w_{x y}[a, \alpha, \gamma] X[\alpha] Y[\gamma]+ \\
& +\sum_{\beta \in O_{v}[a]} \sum_{\gamma \in O_{y}[a]} w_{v y}[a, \beta, \gamma] V[\beta] Y[\gamma]+\sum_{\alpha \in O_{x}[a]} \sum_{\beta \in O_{v}[a]} \sum_{\gamma \in O_{y}[a]} w_{x v y}[a, \alpha, \beta, \gamma] X[\alpha] V[\beta] Y[\gamma]=0,
\end{aligned}
$$

where $X[a] \in R^{n}, V[a] \in R^{m}, Y[a] \in R^{l}$ - state, control and information in the system node; $w_{x}[a, \alpha] \in R^{c \times n}$, $w_{v}[a, \beta] \in R^{c \times m}, \quad w_{y}[a, \gamma] \in R^{c \times l}, \quad w_{x v}[a, \alpha, \beta] \in R^{c \times n \times m}, w_{x y}[a, \alpha, \gamma] \in R^{c \times n \times l}, \quad w_{v y}[a, \beta, \gamma] \in R^{c \times m \times l}$, $w_{x v y}[a, \alpha, \beta, \gamma] \in R^{c \times n \times m \times l}$ - matrix parameters; $O_{x}[a], O_{v}[a], O_{y}[a]$ - neighborhoods of a's node on the state, management and information adequately; $a, \alpha, \beta, \gamma \in A, A=\left\{a_{1}, a_{2}, \ldots, a_{n}\right\}$ - a finite set of system components.

Model (1) with a detailed record takes the following form:

$$
\left\{\begin{array}{l}
w_{x}[1,1] \cdot x[1]+w_{x}[1,2] \cdot x[2]+\ldots+w_{x v y}[1,2,1,1] \cdot x[2] \cdot v[1] \cdot y[1]+ \\
+w_{x v y}[1,2,2,1] \cdot x[2] \cdot v[1] \cdot y[2]=0 \\
\cdots \\
w_{x}[3,2] \cdot x[2]+w_{x}[3,3] \cdot x[3]+\ldots+w_{x v y}[3,3,2,3] \cdot x[3] \cdot v[3] \cdot y[2]+ \\
+w_{x v y}[3,3,3,3] \cdot x[3] \cdot v[3] \cdot y[3]=0 .
\end{array}\right.
$$

\begin{tabular}{|c|c|}
\hline $\mathrm{x}[1]$ & Coiling temperature for the first area of the site strip, ${ }^{\circ}$ \\
\hline $\mathrm{x}[2]$ & Coiling temperature for the second area of the site strip, ${ }^{\circ}$ \\
\hline$x[3]$ & Coiling temperature for the third area of the site strip, ${ }^{\circ}$ \\
\hline $\mathrm{v}[1]$ & The amount of included half-sections of water cooling for the first area of the site strip \\
\hline $\mathrm{v}[2]$ & The amount of included half-sections of water cooling for the second area of the site strip \\
\hline $\mathrm{v}[3]$ & The amount of included half-sections of water cooling for the third area of the site strip \\
\hline$y[1]$ & Cooling water temperature, ${ }^{\circ}$ \\
\hline $\mathrm{y}[2]$ & Cooling water temperature, ${ }^{\circ}$ \\
\hline $\mathrm{y}[3]$ & Cooling water temperature, ${ }^{\circ}$ \\
\hline
\end{tabular}

Essential components of $\mathrm{x}$ state, $\mathrm{v}$ management, and the third parameter, for which the $\mathrm{y}$ information may be taken (Table 1) were distinguish for the model.

Table 1. Components of the state, control and information.

Let's state components' values of state, management and information and conduct trilinear neighborhood model's identification based on previously developed algorithms. State's value a according to technological parameters:

$$
x[1]=587^{\circ} ; x[2]=573^{\circ} ; x[3]=587^{\circ} \text {. }
$$

Management's value a according to technological parameters:

$\mathrm{v}[1]=15$ piece; $\mathrm{v}[2]=28$ piece; $\mathrm{v}[3]=18$ piece.

Information's value a according to technological parameters:

$$
\mathrm{y}[1]=\mathrm{y}[2]=\mathrm{y}[3]=40^{\circ} \mathrm{C} \text {. }
$$


Due to the different order of the data's input the normalization according to the formula is produce:

$$
x^{\prime}=\frac{x-\bar{x}}{\sigma}
$$

where $\mathrm{x}$ - normalized value, $\bar{x}$ - arithmetic mean, $\sigma$ - values' root-mean-square deviation. After the normalization we get:

$$
\begin{aligned}
& \mathrm{x}[1]=0,70711 ; \mathrm{x}[2]=-1,41421 ; \mathrm{x}[3]=0,70711 ; \\
& \mathrm{v}[1]=-0,95962 ; \mathrm{v}[2]=1,37945 ; \mathrm{v}[3]=-0,41983 ; \\
& \mathrm{y}[1]=\mathrm{y}[2]=\mathrm{y}[3]=1 .
\end{aligned}
$$

We'll get that $w y[1,1]=w y[2,2]=w y[3,3]=1$. As a result of the identification we obtain:

$$
\mathrm{wx}[3,2]=0.072, \mathrm{wx}[3,3]=-0.036, \ldots, \mathrm{wxvy}[3,3,2,3]=-0.05, \operatorname{wxvy}[3,3,3,3]=0.015 \text {. }
$$

Let's express neighborhood variables $\mathrm{x}, \mathrm{v}$ and $\mathrm{y}$ through parametric variable $\mathrm{U}$. Linear dependence are as follows:

$$
\begin{aligned}
& x[1]=x[3]=0.0015 \cdot U+1.1617 ; x[2]=0.0015 \cdot U-1.8688 \\
& v[1]=v[2]=v[3]=0.0072 \cdot U+3.5386 \\
& y[1]=y[2]=y[3]=0.0005 \cdot U+1 .
\end{aligned}
$$

Substituting given dependencies into the system (1) and summing the equation, we get a general parametric equation of neighborhood model:

$$
Z=-0,00285 \cdot U+1,11056 \cdot 10^{-7} \cdot U^{2}+2,5266 \cdot 10^{-10} \cdot U^{3}-0,0259 .
$$

\section{RESULTS AND ANALYSIS}

In this section, it is explained the results of research and at the same time is given the comprehensive discussion. Results can be presented in figures, graphs, tables and others that make the reader understand easily [1]-[4]. The discussion can be made in several sub-chapters.

On the analogy of (4), let's examine the function

$$
V_{a, b}(x)=x^{3}+a \cdot x^{2}+b \cdot x
$$

Whose first and second derivative looks like the following:

$$
\begin{aligned}
& \frac{d V}{d x}=3 \cdot x^{2}+2 a \cdot x+b=0, \\
& \frac{d^{2} V}{d x^{2}}=6 \cdot x+2 a=0 .
\end{aligned}
$$

Excluding $\mathrm{x}$ from the equation (6) and (7), we'll get parabola equation:

$$
b=\frac{1}{3} a^{2}
$$

On condition ${ }^{b<\frac{1}{3} \cdot a^{2}}$, the function (5) has two critical points critical points such as minimum and maximum, and on condition $b>\frac{1}{3} \cdot a^{2}$ - critical points are absent. 
Here is a general parametric equation (4) to the standard form:

$$
Z=U^{3}+439,547 \cdot U^{2}-1,13 \cdot 10^{7} \cdot U-1,025 \cdot 10^{8}
$$

The condition $b<\frac{1}{3} \cdot a^{2}$ is realized, i.e. $\quad-1,13 \cdot 10^{7}<\frac{1}{3} \cdot(439,547)^{2}=64400,522$. That's why the function (9) has one minimum point $U=1799,7$ and one maximum point $U=-2092,8$. At the reverse transition from the parametric variable to the neighborhood one, substituting the $U$ value, which corresponds to minimum and maximum values, $\mathrm{x}, \mathrm{v}$ and $\mathrm{y}$ are beyond the permissible values. This means that when operating in the positions of stable (minimum) or unstable (maximum) balance, we'll get the metal of unsatisfactory quality at the output.

Thus, we can conclude that the system is in an area in which it is impossible to say with certainty about the stability of the system. Working that way, coefficients' values of the general parametric equation may change, which entails the risk of reverse conditions that, according to [5], says about the loss of the equilibrium position and the possibility of the system's conversion to a new state.

\section{CONCLUSION}

In this paper the mathematical trilinear neighborhood model of temperature's formation of hot rolled strip's coiling is obtained, the technique of determining the composition of extremums according to the function's coefficients is presented. A hypothesis of the system's areas of stability is suggested. The developed mathematical neighborhood model can take into account other technological parameters of the rolling process and steel's chemical composition.

\section{REFERENCES}

[1] A. M. Shmyrin and I. A. Sedykh, "Identification and Control Algorithms of Functioning for Neighborhood Systems Based on Petri Nets," Automation and Remote Control, vol/issue: 71(6), pp. 1265-1274, 2010.

[2] S. L. Blyumin, et al., "Bilinear Neighborhood Systems," Monograph. Lipetsk, Lipetsk State Technical University Publishers, pp. 131, 2006.

[3] A. L. Genkin and I. V. Nikulin, "The Use of Simulation in the Control Sheet-Rolling Complex," Informacionnye Tehnologii i Vychislitel'nye Sistemy, vol. 2, pp. 75-79, 2011.

[4] A. M. Korneev, et al., "Criterion for Assessing The Effectiveness of Functioning of Complex Systems Based on The Use The Amount of Information," International Journal of Applied Engineering Research, vol/issue: 10(11), pp. 27961-27980, 2015.

[5] J. K. Alekseev and A. P. Sukhorukov, "Introduction to the Theory of Catastrophes," Librokom, pp. 176, 2013. 